\title{
SEARCHING SHEAR FORCES FLOWS FOR AN ARBITRARY CROSS-SECTION OF A THIN-WALLED BAR: DEVELOPMENT OF NUMERICAL ALGORITHM BASED ON THE GRAPH THEORY
}

\author{
Vitalina $V$. Yurchenko \\ Kyiv National University of Civil Engineering and Architecture, Kyiv, UKRAINE
}

\begin{abstract}
Searching problem of shear stresses on outside longitudinal edges of an arbitrary cross-section (including open-closed multi-contour cross-sections) of a thin-walled bar subjected to the general load case has been considered in the paper. Detail numerical algorithm intended to solve the formulated problem using mathematical apparatus of the graph theory has been proposed by the paper. The algorithm is oriented on software implementation in systems of computer-aided design of thin-walled bar structures.
\end{abstract}

Keywords: thin-walled bar, an arbitrary cross-section, shear forces flow, closed contour, graph theory

\section{ПОИСК ПОТОКОВ КАСАТЕЛЬНЫХ УСИЛИЙ ДЛЯ ПРОИЗВОЛЬНОГО СЕЧЕНИЯ ТОНКОСТЕННОГО СТЕРЖНЯ: РАЗРАБОТКА ЧИСЛЕННОГО АЛГОРИТМА НА ОСНОВЕ ТЕОРИИ ГРАФОВ}

\author{
B.В. Юрченко \\ Киевский национальный университет строительства и архитектуры, г. Киев, УКРАИНА
}

\begin{abstract}
Аннотация: Рассмотрена задача поиска значений потоков касательных усилий для произвольного сечения (открыто-замкнутого многоконтурного сечения) тонкостенного стержня для общего случая нагружения. Разработан детальный алгоритм численного решения сформулированной задачи с использованием математического аппарата теории графов, ориентированный на программную реализацию в системах автоматизированного проектирования тонкостенных стержневых систем.
\end{abstract}

Ключевые слова: тонкостенный стержень, произвольное сечение, потоки касательных усилий, замкнутый контур, теория графов

\section{INTRODUCTION}

Let us consider the problem of shear stresses on longitudinal edges of an arbitrary section of a thin-walled bar that can consist of several closed (connected and/or disconnected) contours and/or also open parts. This problem has been studied by V. I. Slivker [1, 2, 8] for the general loading case of the thin-walled bar.

Further investigation in this area requires development of detail algorithm intended to software implementation in a computer-aided design system for thin-walled bar structures. It is reasonable to construct this algorithm using mathematical apparatus of the graph theory [11]. The graph theory has been applied in $[7,10,12]$ for calculation the geometrical sectional properties of thin-walled bars with hybrid (open-closed) types of cross-sections. The graph theory has been also used to analysis of thinwalled bars with multi-contour cross-sections by G. Alfano in [6] and A. Prokić, A [10]. Herewith, the problem of the contoured distribution of shear forces flows for hybrid (including multi-contour, open-closed) thinwalled sections was out of consideration. 


\section{INITIAL DATA AND SYSTEMS OF COORDINATES}

Let us introduce on the plane of thin-walled section the Descartes rectangular system of coordinates $y \mathrm{Oz}$ with origin of the system placed in center of mass of the section (Fig. 1.1), direction of the coordinate system axes coincides with direction of principle axes of inertia $u O v$.

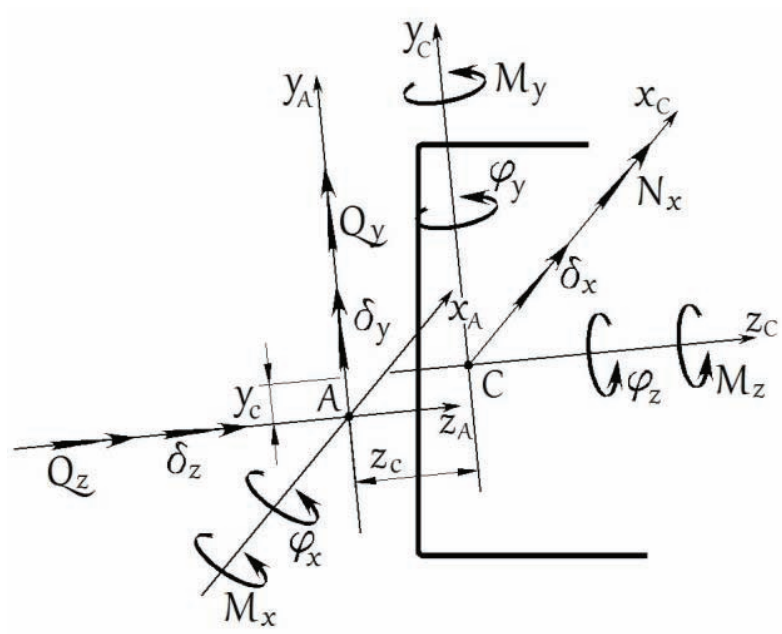

Figure 1.1. Cross-section of a thin-walled bar with indication of positive directions of forces and moments.

Let us introduce in further consideration the system of angular position coordinate with origin of such system in certain (in general case randomly selected) sectional point. Each considered sectional point can be associated with angular position $\varsigma$. The value $\varsigma$ should be calculated as geometrical length of the curve which is constructed from the origin to the considered sectional point taken along sectional contour. We also assume that increment of the angular position $\varsigma$ corresponds to the positive direction of section path tracing.

We assume that integral geometrical properties of the section are known: $A$ - cross-sectional area, $I_{y}$ and $I_{z}$ - second moments of area relating to the main axes of inertia which coincide with axes of global Descartes system of coordinates $y \mathrm{Oz} ; \mathrm{I}_{\omega}$ - sectorial moment of inertia; $I_{t}-$ second moment of area for pure torsion. We also assume that elasticity module $E$ and shear module $G$ are constants for the whole section of the thin-walled bar.

In general case a thin-walled bar is subjected to the action of eight force factors. Axial force $N$, bending moments $M_{y}$ and $M_{z}$ relating to the principle axes of inertia and bi-moment $B$ are applied at the center of mass $C$ (see Fig. 1.1) of the section and caused normal stresses in section $\sigma_{i}(x, \varsigma)$ :

$$
\begin{aligned}
& \sigma_{i}(x, \varsigma)=\frac{N(x)}{A}+\frac{M_{y}(x)}{I_{y}} z_{i}(\varsigma)+ \\
& +\frac{M_{z}(x)}{I_{z}} y_{i}(\varsigma)+\frac{B(x)}{I_{\sigma}} \varpi_{i}(\varsigma)
\end{aligned}
$$

where $y_{i}(\varsigma), z_{i}(\varsigma), \varpi_{i}(\varsigma)-$ coordinates and sectorial coordinate of the considered point in cross-section of a thin-walled bar.

Shear forces $Q_{y}$ and $Q_{z}$, free torsion moment $M_{x}$ and moment of restrained torsion $M_{\omega}$ are applied at the shear center $A$ of the section (Fig. 1.1) and caused shear stresses in crosssection, which can be written in terms of shear forces flows $T_{j}(x, \varsigma)$ as presented below:

$$
\tau_{j}(x, \varsigma)=\frac{T_{j}(x, \varsigma)}{\delta_{j}(\varsigma)}
$$

where $\delta_{j}(\varsigma)-$ thickness of considered $j^{\text {th }}$ section element.

An arbitrary section of a thin-walled bar can be described by the set of sectional points

$$
\mathbf{P}=\left\{\vec{p}_{p}=\left\{y_{p}, z_{p}\right\} \mid p=\overline{1, n_{p}}\right\}
$$

$\left(y_{p}\right.$ and $z_{p}-$ coordinates of $p^{\text {th }}$ sectional point in global Descartes coordinate system $y O z$ ) and by the set of sectional segments 


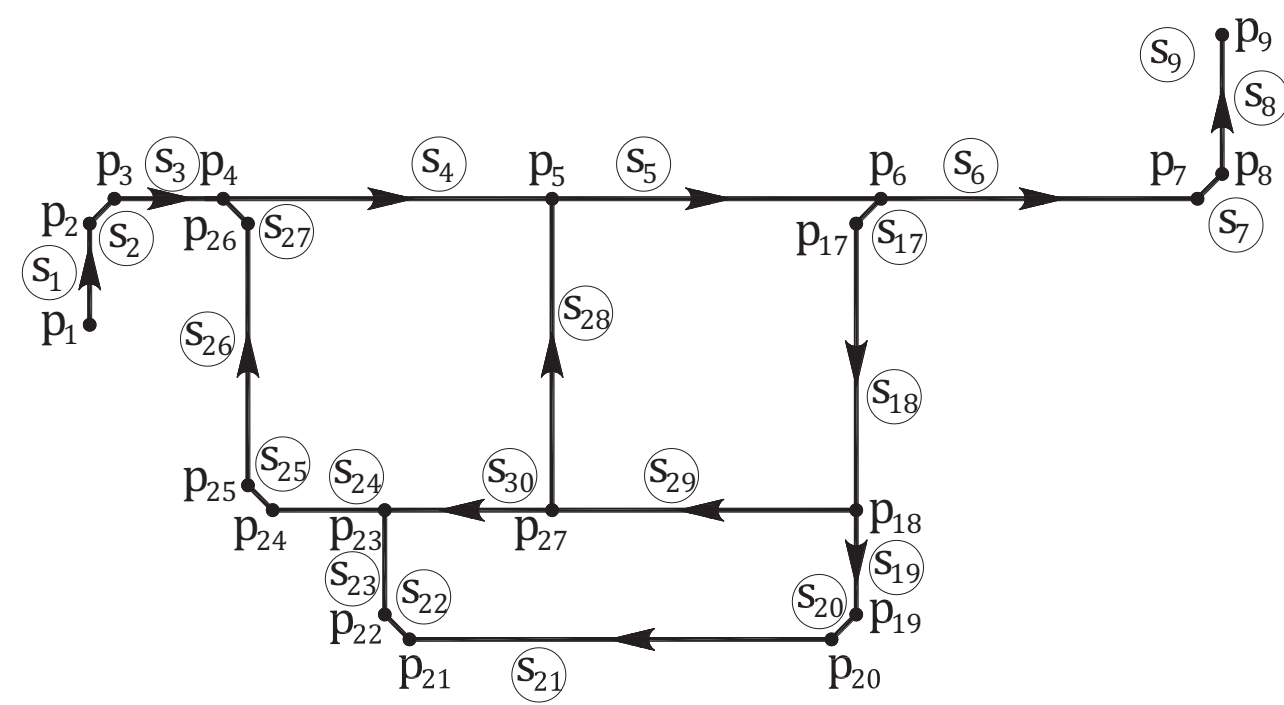

\section{Figure 1.2. Arbitrary cross-section of a thin-walled bar determined on the set of sectional points $\mathbf{P}$ and set of sectional segments $\mathbf{S}$.}

$$
\mathbf{S}=\left\{\vec{s}_{s}=\left\{p_{s}^{s t}, p_{s}^{\text {end }}\right\} \mid s=\overline{1, n_{s}}\right\}
$$

which connect some two sectional points (Fig. 1.2), where $n_{p}$ and $n_{s}-$ numbers of sectional points and segments accordingly. Specified segment thickness

$$
\boldsymbol{\delta}=\left\{\delta_{s} \mid s=\overline{1, n_{s}}\right\}
$$

corresponds to each sectional segment.

The set of sectorial coordinates

$$
\boldsymbol{\omega}=\left\{\omega_{p} \mid p=\overline{1, n_{p}}\right\}
$$

and the set of normalized sectorial coordinates

$$
\varpi=\left\{\varpi_{p} \mid p=\overline{1, n_{p}}\right\}
$$

of the section correspond with the set of sectional points $\mathbf{P}$, assuming that the values of the sectorial coordinates and normalized sectorial coordinates in each cross-sectional point are known [4].

The set of angular positions is actually intended to implement numerical integration taken along thin-walled section contour (for example, when calculating geometrical properties of the cross-section, values of shear forces flows, etc.), where $\kappa-$ ordinal number of segment, $n_{\varsigma}-1-$ number of sectional segments. It should be noted that an angular positions are attributes of the ends of sectional segments.

Initial data about a thin-walled section should be mapped onto the set of angular positions

$$
\varsigma, \kappa=\overline{1, n_{\varsigma}-1}
$$

by means of formation corresponded sets of sectional segments

$$
\mathbf{S}^{\varsigma}=\left\{\vec{S}_{\kappa}^{\varsigma}=\left\{\varsigma_{\kappa}^{\text {start }}, \varsigma_{\kappa}^{\text {end }}\right\}: \varsigma_{\kappa}^{\text {start }}, \varsigma_{\kappa}^{\text {end }} \subseteq \varsigma\right\},
$$

set of sectorial coordinates

$$
\boldsymbol{\omega}^{\varsigma}=\left\{\vec{\omega}_{\kappa}^{\varsigma}=\left\{\omega_{\kappa}^{\text {start }}, \omega_{\kappa}^{\text {end }}\right\}: \omega_{\kappa}^{\text {start }}, \omega_{\kappa}^{\text {end }} \subseteq \boldsymbol{\omega}\right\}
$$

for ends of sectional segments as well as the set 
of thicknesses

$$
\boldsymbol{\delta}^{\varsigma}=\left\{\delta_{\kappa}^{\varsigma} \subseteq \boldsymbol{\delta}\right\}
$$

for segments, $\kappa=\overline{1, n_{\varsigma}-1}$.

\section{DISTRIBUTION OF SHEAR FORCES FLOWS TAKEN ALONG CLOSED CONTOURS OF AN ARBITRARY CROSS-SECTION OF THIN-WALLED BAR}

\subsection{Construction of connected graph} $\mathbf{G}=\{\mathbf{V}, \mathbf{R}\}$ associated with a section of thinwalled bar.

An arbitrary cross-section of thin-walled bar can be associated with planar connected nonoriented graph $\mathbf{G}$ determined on the sets of $\mathbf{G}=\{\mathbf{V}, \mathbf{R}\}$, where $\mathbf{V}$ - finite set of graph vertices, $\mathbf{R}$ - the set of graph edges or the set of unordered pairs on $\mathbf{V}$ (see Fig. 2.1) [9]. Herewith, for each graph edge

$$
\mathbf{r}=\{u, v\} \in \mathbf{R}
$$

we assume that $u \neq v$.

Vertices of the graph $\mathbf{G}^{\boldsymbol{G}}$ are associated with characteristic sectional points only, which are:

1) branch points - sectional points connected with more than two sectional segments,

$$
\mathbf{v}^{\mathbf{p}}=\left\{\vec{p}_{v} \mid v=\overline{1, n_{v}}\right\},
$$

here $n_{v}$ - number of these points;

2) end points - sectional points connected with only one sectional segment

$$
\mathbf{v}_{\text {end }}^{\mathbf{p}}=\left\{\vec{p}_{g} \mid g=\overline{1, n_{g}}\right\},
$$

here $n_{g}$ - number of these points.

Edges of graph $\mathbf{G}$ are associated with sectional parts located between characteristic sectional points (with unbranched sectional parts).

An edge of the graph $\mathbf{G}$, as a rule, may contain several sectional segments, so full information about edge $\mathbf{R}_{j}^{\varsigma}$ of the graph can be described by the set of sectional segments

$$
\vec{s}_{r}^{\varsigma}, r=\overline{1, n_{\varsigma r j}},
$$

from the array

$$
\mathbf{S}^{\varsigma}=\left\{\vec{s}_{\kappa}^{\varsigma}=\left\{\varsigma_{\kappa}^{\text {start }}, \varsigma_{\kappa}^{\text {end }}\right\} \mid \kappa=\overline{1, n_{\varsigma}-1}\right\}, \vec{s}_{r}^{\varsigma} \in \mathbf{S}^{\varsigma},
$$

belonged to considered graph edge, $\vec{s}_{r}^{\varsigma} \in \mathbf{R}_{j}$ :

$$
\mathbf{R}_{j}^{\varsigma}=\left\{\vec{s}_{r}^{\varsigma}: \vec{s}_{r}^{\varsigma} \in \mathbf{S}^{\varsigma} \wedge \vec{s}_{r}^{\varsigma} \in \mathbf{R}_{j} \mid r=\overline{1, n_{\varsigma r j}}\right\},
$$

here $n_{c r j}-$ number of segments for $j^{\text {th }}$ graph edge. The set of all graph edges defined on the set of segments $\mathbf{S}^{\varsigma}$ can be expressed as

$$
\mathbf{R}^{\varsigma}=\left\{\mathbf{R}_{j}^{\varsigma} \mid j=\overline{1, n_{r}}\right\} .
$$

We also assume that an arbitrary section of thinwalled bar may contain some quantity of closed contours. Each closed section contour is associated with cycle of graph $\mathbf{G}^{\boldsymbol{r}}$ or with vertices cortege $v_{0}^{k}, v_{1}^{k}, v_{2}^{k}, \ldots, v_{n}^{k}$, such that

$$
v_{i}^{k} \mapsto v_{i+1}^{k} \forall i \Leftrightarrow \exists v_{i+1}^{k},
$$

where $n_{k}-$ number of closed contours in section (number of graph $\mathbf{G}$ cycles).

Some closed section contour $\boldsymbol{\Gamma}_{k}^{r_{S}}$ (basic cycle of the graph $\mathbf{G}$ ) can be definitely determined by the set of graph edges $\mathbf{R}_{j}^{\varsigma} \in \mathbf{R}^{\varsigma}$ belonged to the considered contour

$$
\Gamma_{k}^{r \varsigma}=\left\{\mathbf{R}_{j}^{\varsigma} \mid j=\overline{1, n_{r \varsigma \Gamma_{k}}}\right\}
$$

where $n_{r_{\varsigma} \Gamma_{k}}-$ number of graph edges belonged to $k^{\text {th }}$ closed section contour. 


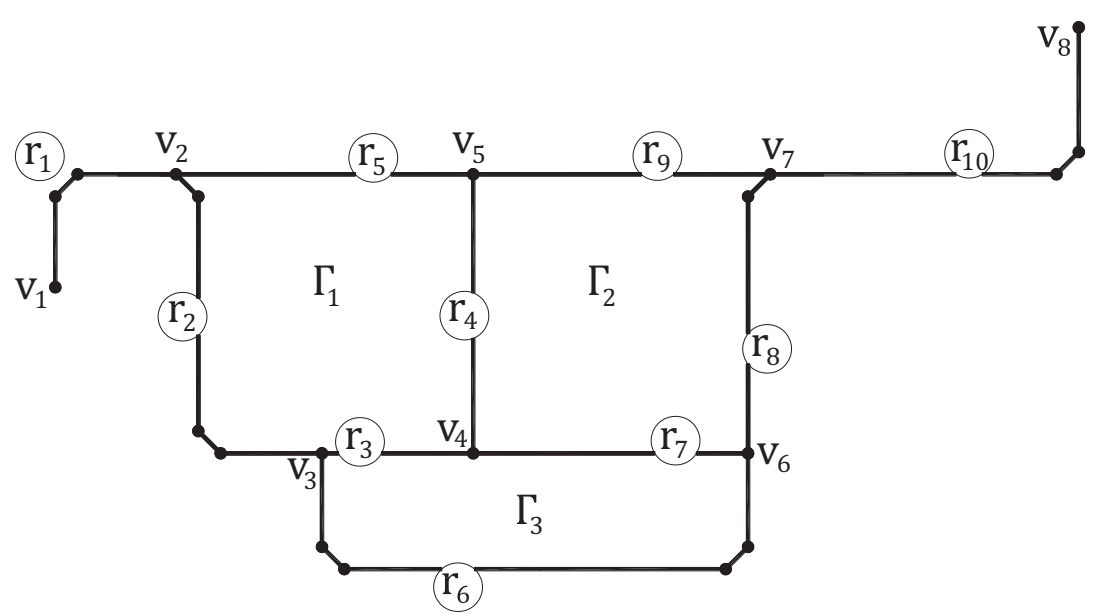

Figure 2.1. Graph $\mathbf{G}=\{\mathbf{V}, \mathbf{R}\}$ associated with cross-section of thin-walled bar (graph vertices $-v_{1}, v_{2} \ldots v_{10}$, graph edges $\left.-r_{1}, r_{2} \ldots r_{13}\right)$.

Besides, it's convenient to have the mapping of closed section contour $\Gamma_{k}^{r \varsigma}$ onto the set of sectional segments

$$
\vec{s}_{m}^{\varsigma}, \vec{s}_{m}^{\varsigma} \in \mathbf{S}^{\varsigma}
$$

belonged to considered closed contour, $\forall m=\overline{1, n_{\varsigma \Gamma_{k}}}$ :

$$
\boldsymbol{\Gamma}_{k}^{\varsigma}=\left\{\vec{s}_{m}^{\varsigma}:\left[\begin{array}{c}
\vec{s}_{m}^{\varsigma} \in \mathbf{S}^{\varsigma} \\
\exists \mathbf{R}_{\alpha}^{\varsigma} \subseteq \mathbf{R}^{\varsigma}: \vec{s}_{m}^{\varsigma} \subseteq \mathbf{R}_{\alpha}^{\varsigma} \wedge \mathbf{R}_{\alpha}^{\varsigma} \subseteq \boldsymbol{\Gamma}_{k}^{r \varsigma}
\end{array}\right\}\right.
$$

here $n_{\varsigma \Gamma_{k}}-$ number of sectional segments belonged to $k^{\text {th }}$ closed section contour.

Closed section contours (basic cycles of the graph $\mathbf{G}$ ) defined on the set of graph edges $\mathbf{R}^{\varsigma}$ and on the set of section segments $\mathbf{S}^{\boldsymbol{\varsigma}}$ can be described as

$$
\boldsymbol{\Phi}^{r \varsigma}=\left\{\boldsymbol{\Gamma}_{k}^{r \varsigma} \mid k=\overline{1, n_{k}}\right\}
$$

and

$$
\boldsymbol{\Phi}^{\varsigma}=\left\{\boldsymbol{\Gamma}_{k}^{\varsigma} \mid k=\overline{1, n_{k}}\right\}
$$

accordingly. It should be noted that identification of closed contours in the section
$\boldsymbol{\Phi}^{r \varsigma}$ and $\boldsymbol{\Phi}^{\varsigma}$ can be easily implemented using depth-first search algorithms on the graph.

Let us compose incidence matrix $\dot{\mathbf{I}}$ for graph $\mathbf{G}$ with dimensions $n_{v} \times n_{r}$,

$$
\dot{\mathbf{I}}=\left\{g_{i j} \mid i=\overline{1, n_{v}}, j=\overline{1, n_{r}}\right\} .
$$

Components of the matrix take on following values: $g_{i j}=1-$ if $i^{\text {th }}$ graph vertex is start vertex for $j^{\text {th }}$ edge; $g_{i j}=-1-$ if $i^{\text {th }}$ graph vertex is end vertex for $j^{\text {th }}$ edge; $g_{i j}=0-$ otherwise. Let us also introduce in further consideration matrix

$$
|\dot{\mathbf{I}}|=\left\{\left|g_{i j}\right| \mid i=\overline{1, n_{v}}, j=\overline{1, n_{r}}\right\}
$$

composed using modulus of elements $g_{i j}$ of matrix $\dot{\mathbf{I}}$.

Next, we can compose the matrix of basic graph cycles $\mathbf{F}$ with dimension $n_{k} \times n_{r}$,

$$
\mathbf{F}=\left\{f_{k j}\right\}, k=\overline{1, n_{k}}, j=\overline{1, n}_{r} .
$$

Components of the matrix take on following values: $f_{k j}=1-$ if $j^{\text {th }}$ graph edge belongs to $k^{\text {th }}$ basic graph cycle $\left(\mathbf{R}_{j}^{\varsigma} \subseteq \boldsymbol{\Gamma}_{k}^{\varsigma}\right)$ and edge direction coincides with the positive direction of path 
tracing; $f_{k j}=-1-$ if $j^{\text {th }}$ graph edge belongs to $k^{\text {th }}$ basic graph cycle $\left(\mathbf{R}_{j}^{\varsigma} \subseteq \Gamma_{k}^{\varsigma}\right)$ and edge direction does not coincide with positive direction of path tracing; $f_{k j}=0-$ if $j^{\text {th }}$ graph edge does not belong to $k^{\text {th }}$ basic graph cycle $\left(\mathbf{R}_{j}^{\varsigma} \cap \boldsymbol{\Gamma}_{k}^{\varsigma}=\varnothing\right)$.

\subsection{Distribution of the shear forces flows} taken along closed section contours

Each $j^{\text {th }}$ edge $\mathbf{R}_{j}^{\varsigma}, \quad j=\overline{1, n_{r}}$ of graph $\mathbf{G}$ corresponds with a constant - edge weight, $\forall \kappa: \vec{s}_{\kappa}^{\varsigma} \in \mathbf{R}_{j}^{\varsigma} \wedge \vec{s}_{\kappa}^{\varsigma} \in \mathbf{S}^{\varsigma}:$

$$
\begin{aligned}
& p_{j}=\int_{\ell_{r j}} \frac{d \varsigma}{\delta(\varsigma)}=\sum_{r=1}^{n_{\zeta r j}} \int_{\ell_{\varsigma} \in \mathbf{R}_{j}^{\zeta}} \frac{d \varsigma}{\delta(\varsigma)}= \\
& =\sum_{r=1}^{n_{\zeta r j}} \frac{1}{\delta_{\kappa}^{\varsigma}} \int_{\varsigma_{\kappa}}^{\varsigma_{\kappa+1}} d \varsigma=\sum_{r=1}^{n_{\zeta r j}} \frac{l_{\kappa}^{\varsigma}}{\delta_{\kappa}^{\varsigma}} .
\end{aligned}
$$

Let us also compose weighting matrix of unbranched sectional parts (edges of graph $\mathbf{G}$ ) square matrix $\mathbf{W}$ with dimensions $n_{r} \times n_{r}$ and diagonal elements $p_{j}, j=\overline{1, n_{r}}$ :

$$
\begin{aligned}
& \mathbf{W}=\left[\begin{array}{cccc}
p_{1} & 0 & \ldots & 0 \\
0 & p_{2} & \ldots & 0 \\
\vdots & \vdots & \ddots & \vdots \\
0 & 0 & 0 & p_{n_{r}}
\end{array}\right] \quad \begin{array}{c}
\text { (2.2) } \\
\text { factors } \tilde{a}_{k}, k=\overline{1, n_{k}} \text {, of shermulated in [2] and pres } \\
\text { formulars }
\end{array} \\
& {\left[\begin{array}{ccccccc}
\tilde{p}_{11} & -p_{12} & \cdots & -p_{1 k} & \cdots & -p_{1 n_{k}} & \Omega_{1} \\
-p_{21} & \tilde{p}_{22} & \cdots & -p_{2 k} & \cdots & -p_{2 n_{k}} & \Omega_{2} \\
\vdots & \vdots & \ddots & \vdots & \vdots & \vdots & \vdots \\
-p_{k 1} & -p_{k 2} & \cdots & \tilde{p}_{k k} & \cdots & -p_{k n_{k}} & \Omega_{k} \\
\vdots & \vdots & \vdots & \vdots & \ddots & \vdots & \vdots \\
-p_{n_{k} 1} & -p_{n_{k} 2} & \cdots & -p_{n_{k}} & \cdots & \tilde{p}_{n_{k} n_{k}} & \Omega_{n_{k}} \\
\Omega_{1} & \Omega_{2} & \cdots & \Omega_{k} & \cdots & \Omega_{n_{k}} & 0
\end{array}\right] \times\left[\begin{array}{c}
\tilde{a}_{1} \\
\tilde{a}_{2} \\
\vdots \\
\tilde{a}_{k} \\
\vdots \\
\tilde{a}_{n_{k}} \\
\lambda
\end{array}\right]=\left[\begin{array}{c}
0 \\
0 \\
\vdots \\
0 \\
\vdots \\
0 \\
\Omega_{0}
\end{array}\right] ; }
\end{aligned}
$$

Besides, each $j^{\text {th }}$ graph edge $\mathbf{R}_{j}^{\varsigma}$ corresponds with the increment of sectorial coordinate

$$
\begin{array}{r}
\Delta \omega_{\mathbf{r}}^{\varsigma}=\left\{\Delta \omega_{r, j}^{\varsigma} \mid j=\overline{1, n_{r}}\right\}^{T}, \\
\forall \kappa: \vec{S}_{\kappa}^{\varsigma} \in \mathbf{R}_{j}^{\varsigma} \wedge \vec{S}_{\kappa}^{\varsigma} \in \mathbf{S}^{\varsigma}: \\
\Delta \omega_{r, j}^{\varsigma}=\int_{\ell_{r j}} \rho d \varsigma=\int_{\ell_{r j}} d \omega=\sum_{r=1}^{n_{\zeta \zeta j}} \int_{\ell_{\varsigma} \in \mathbf{R}_{j}^{\varsigma}} d \omega= \\
=\sum_{r=1}^{n_{\zeta r}} \int_{\varsigma_{\kappa}}^{\varsigma_{\kappa+1}} d \omega=\sum_{r=1}^{n_{\zeta r i j}} \Delta \omega_{\kappa}^{\varsigma} .
\end{array}
$$

Each closed contour of the section $\Gamma_{k}^{r \varsigma}$, $k=\overline{1, n_{k}}$, corresponds to the following constant - contour weight, $f_{k j} \in \mathbf{F}, \forall j: \mathbf{R}_{j}^{\varsigma} \subseteq \Gamma_{k}^{r \varsigma}$ :

$$
\tilde{p}_{k}=\int_{\Gamma_{k}^{r \zeta \zeta}} \frac{d \varsigma}{\delta(\varsigma)}=\int_{\mathbf{R}_{j}^{\zeta} \subseteq \Gamma_{k}^{r \zeta}} \frac{d \varsigma}{\delta(\varsigma)}=\sum_{j=1}^{n_{r \zeta \zeta k}}\left|f_{k j}\right| p_{j} .
$$

Let us consider the problem of free torsion for an arbitrary thin-walled section which consists of certain number of closed (connected and/or disconnected) contours as well as of open parts. In general case the following resolving system of equation for calculation the distribution factors $\tilde{a}_{k}, k=\overline{1, n_{k}}$, of shear forces flows taken along closed contours of the section has been formulated in [2] and presented below: 
here diagonal elements of the matrix are weights of $k^{\text {th }}$ closed contour,

$$
\tilde{p}_{k k}=\tilde{p}_{k}, k=\overline{1, n_{k}}
$$

$\Omega_{k}-$ double area embraced by $k^{\text {th }}$ closed contour $\Gamma_{k}^{\varsigma}$,

$$
\Omega_{0}=\sum_{k=1}^{n_{k}} \Omega_{k}
$$

Other elements of matrix $p_{\alpha \beta}$ take on zero value $p_{\alpha \beta}=p_{\beta \alpha}=0$, when corresponded closed contours have no common edges:

$$
\Gamma_{\alpha}^{\varsigma} \cap \Gamma_{\beta}^{\varsigma}=\varnothing
$$

and the sum value of weights for all common edges [3]:

$$
p_{\alpha \beta}=p_{\beta \alpha}=\sum_{r} p_{r}, \forall r: \mathbf{R}_{r}^{\varsigma} \subseteq \boldsymbol{\Gamma}_{\alpha}^{\varsigma} \wedge \mathbf{R}_{r}^{\varsigma} \subseteq \boldsymbol{\Gamma}_{\beta}^{\varsigma} .
$$

Solution of the system of algebraic equations returns the column vector of factors

$$
\mathbf{A}_{k}=\left\{\tilde{a}_{k} \mid k=\overline{1, n_{k}}\right\}
$$

for distribution of shear forces flows taken along closed contours of the section. Based on $\mathbf{A}_{k}$ we can generate the column vector of factors for distribution of shear forces flows taken along graph $\mathbf{G}$ edges:

$$
\mathbf{A}_{r}=\left\{a_{j} \mid j=\overline{1, n_{r}}\right\},
$$

where each element should be determined as:

$$
a_{j}=\sum_{k=1}^{n_{k}} f_{k j} \bar{a}_{k}, f_{k j} \in \mathbf{F}, \forall j=\overline{1, n_{r}} .
$$

Since each graph edge $\mathbf{R}_{j}^{\varsigma}, \quad j=\overline{1, n_{r}}$, is described by the set of sectional segments $\vec{s}_{r}^{\varsigma} \in \mathbf{S}^{\varsigma}$ as:

$$
\mathbf{R}_{j}^{\varsigma}=\left\{\vec{s}_{r}^{\varsigma}: \vec{s}_{r}^{\varsigma} \in \mathbf{S}^{\varsigma} \wedge \vec{s}_{r}^{\varsigma} \in \mathbf{R}_{j} \mid r=\overline{1, n_{\varsigma r j}}\right\},
$$

then it is possible to determine for each sectional segment $\vec{s}_{\kappa}^{\varsigma} \in \mathbf{S}^{\varsigma}$ the value of piecewise constant distribution function for shear flows taken along section $a^{\varsigma}(\varsigma)$ as the set of

$$
\mathbf{a}^{\varsigma}=\left\{a_{\kappa}^{\varsigma} \mid \kappa=\overline{1, n_{\varsigma}-1}\right\}
$$

as follows:

$$
a_{\kappa}^{\varsigma}=a_{j}, \forall \kappa: \vec{s}_{\kappa}^{\varsigma} \cap \boldsymbol{\Phi}^{\varsigma} \neq \varnothing,
$$

and

$$
a_{\kappa}^{\varsigma}=0,
$$

otherwise.

\section{RESOLVING EQUATIONS FOR AN ARBITRARY CROSS-SECTION OF THIN-WALLED BAR}

Search problem of shear forces flows for an arbitrary cross-section of thin-walled bar (including open-closed multi-contour crosssections) can be transformed into minimization problem of Castigliano's functional C subject to constraints-equalities of shear forces flows equilibrium formulated for cross-section branch points as well as subject to equilibrium equation for the whole cross-section relating to longitudinal axes of the thin-walled bar [2].

Let present the formulated problem as mathematical programming task, namely as searching of unknown values of shear forces flows at start points of unbranched parts of a section: 


$$
\stackrel{\mathrm{r}}{T_{S}}=\left\{T_{S, j}\right\}^{T}, j=\overline{1, n_{r}},
$$

which ensure the least value of optimum criterion - Castigliano's functional $\mathbf{C}$ :

$$
\mathbf{C}^{*}=\mathbf{C}\left(\vec{T}_{S}^{*}\right)=\min _{T_{s} \in \widetilde{\Im}_{T}} \mathbf{C}\left(\vec{T}_{S}\right)
$$

on hyperplane of feasible decisions $\mathfrak{J}_{T}$ described by the follow system of constraintsequalities:

$$
\left\{\begin{array}{l}
\mathbf{f}\left(\vec{T}_{S}\right)=\left\{f_{v}\left(\vec{T}_{S}\right)=0 \mid v=\overline{1, n_{v}-1}\right\} \\
f_{x}\left(\vec{T}_{S}\right)=0
\end{array}\right.
$$

where $\stackrel{\mathrm{r}}{T_{S}}$ - vector of design variables (searched shear flows); $n_{r}$ - number of unknown shear flows; $\vec{T}_{S}^{*}$ - optimum decision of the problem; $\mathbf{C}^{*}$ - minimum value of Castigliano's functional; $f_{v}$ - function of the vector argument $\stackrel{\mathrm{r}}{T_{S}} ; \quad n_{v}-$ general number of constraintsequalities $f_{v}\left(\vec{T}_{S}\right)$ and $f_{x}\left(\vec{T}_{S}\right)$ which define hyperplane of feasible decisions $\mathfrak{J}_{T}$ in search space.

For Castigliano's functional C in further we will consider Euler's equations only which define strain compatibility conditions and are expressed depending on shear forces flows

$$
\stackrel{\mathrm{r}}{T_{S}}=\left\{T_{S, j}\right\}^{T}, j=\overline{1, n_{r}} .
$$

So it is well known that in case of quadratic functional Euler's equations don't depend on boundary conditions, then kinematic boundary conditions can be accept as homogeneous. As it was shown by V. Slivker in [2, p. 405], Castigliano's functional in this case can be identified with expression for strain energy formulated in terms of stresses as presented below:

$$
\mathbf{C}=\sum_{j=1}^{n_{r}}\left(\int_{1_{j}} \frac{(\sigma(\varsigma))^{2}}{2 E e(\varsigma)} \delta(\varsigma) d \varsigma+\int_{1_{j}} \frac{(\tau(\varsigma))^{2}}{2 G g(\varsigma)} \delta(\varsigma) d \varsigma\right)
$$

or for isotropic material:

$$
\begin{gathered}
\mathbf{C}=\frac{1}{2}\left(\sum_{j=1}^{n_{r}}\left(\int_{1_{j}} \frac{(\sigma(\varsigma))^{2}}{E} \delta(\varsigma) d \varsigma+\int_{1_{j}} \frac{(\tau(\varsigma))^{2}}{G} \delta(\varsigma) d \varsigma\right) ;\right. \\
\mathbf{C}=\frac{1}{2 G}\left(\sum_{j=1}^{n_{r}}\left(\int_{1_{j}} \frac{(\sigma(\varsigma))^{2}}{2(1+v)} \delta(\varsigma) d \varsigma+\int_{1_{j}}(\tau(\varsigma))^{2} \delta(\varsigma) d \varsigma\right) ;\right.
\end{gathered}
$$

Let rewrite Castigliano's functional C (3.4) the dependence from shear forces flows (1.2) as with replacement normal stresses $\sigma(\varsigma)$ by the presented below:

expression (1.1), and shear stresses $\tau(\varsigma)$ - by

$$
\tau_{j}(\varsigma)=\frac{T_{j}(\varsigma)}{\delta_{j}(\varsigma)}=\frac{1}{\delta_{j}(\varsigma)}\left(T_{S, j}-\frac{Q_{z}}{I_{y}} S_{o y, j}(\varsigma)-\frac{Q_{y}}{I_{z}} S_{o z, j}(\varsigma)-\frac{M_{\varpi}}{I_{\varpi}} S_{o \varpi, j}(\varsigma)\right)
$$


Searching Shear Forces Flows for an Arbitrary Cross-Section of a Thin-Walled Bar: Development of Numerical Algorithm Based on the Graph Theory

$$
\begin{aligned}
& \mathbf{C}=\frac{1}{2 G}\left(\left(\sum _ { j = 1 } ^ { n _ { r } } \left(\int_{1_{j}} \frac{1}{2(1+v)}\left(\frac{N}{A}+\frac{M_{y}}{I_{y}} z_{j}+\frac{M_{z}}{I_{z}} y_{j}+\frac{B}{I_{\varpi}} \varpi_{j}\right)^{2} \delta_{j} d \varsigma+\right.\right.\right. \\
& \left.\left.+\int_{1_{j}}\left(T_{S, j}^{2}-2 T_{S, j} \frac{Q_{z}}{I_{y}} S_{o y, j}-2 T_{S, j} \frac{Q_{y}}{I_{z}} S_{o z, j}-2 T_{S, j} \frac{M_{\varpi}}{I_{\varpi}} S_{o \varpi, j}+\left(\frac{Q_{z}}{I_{y}} S_{o y, j}+\frac{Q_{y}}{I_{z}} S_{o z, j}+\frac{M_{\varpi}}{I_{\varpi}} S_{o \varpi, j}\right)^{2}\right) \frac{d \varsigma}{\delta_{j}}\right)\right) .
\end{aligned}
$$

here we omitted functional dependence from angular position $\varsigma$ (to simplify presented formulas):

Let us leave in (3.6) those summands which are dependent from shear forces flows values

$$
\stackrel{\mathrm{r}}{T_{S}}=\left\{T_{S, j}\right\}^{T}, j=\overline{1, n_{r}},
$$

and also denote by the symbol $\mathrm{K}$ all other summands that are don't dependent from the vector $\stackrel{\mathrm{r}}{T_{S}}$. In this way we have obtain expression for Castigliano's functional $\mathbf{C}$ in terms of shear forces flows [2]

$$
\stackrel{\mathrm{r}}{T_{S}}=\left\{T_{S, j}\right\}^{T}
$$

as presented below:

$$
\begin{gathered}
\mathbf{C}=\sum_{j=1}^{n_{r}}\left(\int_{1_{j}}\left(\frac{T_{S, j}^{2}}{2 G}-T_{S, j} \frac{Q_{z}}{G I_{y}} S_{o y, j}-T_{S, j} \frac{Q_{y}}{G I_{z}} S_{o z, j}-T_{S, j} \frac{M_{\varpi}}{G I_{\varpi}} S_{o \varpi, j}\right) \frac{d \varsigma}{\delta_{j}}+\mathrm{K}\right) \\
\mathbf{C}=\sum_{j=1}^{n_{r}}\left(\frac{T_{S, j}^{2}}{2 G} \int_{1_{j}} \frac{d \varsigma}{\delta_{j}}-T_{S, j} \frac{Q_{z}}{G I_{y}} \int_{1_{j}} S_{o y, j} \frac{d \varsigma}{\delta_{j}}-T_{S, j} \frac{Q_{y}}{G I_{z}} \int_{1_{j}} S_{o z, j} \frac{d \varsigma}{\delta_{j}}-T_{S, j} \frac{M_{\varpi}}{G I_{\varpi}} \int_{1_{j}} S_{o \varpi, j} \frac{d \varsigma}{\delta_{j}}+\mathrm{K}\right)
\end{gathered}
$$

where integral

$$
\int_{1_{j}} \frac{d \varsigma}{\delta_{j}}
$$

$$
\begin{aligned}
& S_{h z, j}=\int_{\ell_{r j}} \frac{S_{o z, j}^{\varsigma}(\varsigma) d \varsigma}{\delta(\varsigma)}= \\
& =\sum_{\kappa=1}^{n_{\zeta \zeta j}}\left(\frac{l_{\kappa}^{\varsigma}}{6 \delta_{\kappa}^{\varsigma}}\left(S_{o z, \kappa}^{\varsigma, s t a r t}+4 S_{o z, \kappa}^{\varsigma, \text { mid }}+S_{o z, \kappa}^{\varsigma, \text { end }}\right)\right) ;
\end{aligned}
$$

can be calculated according to (4.5), and integrals

$$
S_{h y, j}=\int_{\ell_{r j}} \frac{S_{o y, j}^{\varsigma}(\varsigma) d \varsigma}{\delta(\varsigma)}=
$$

$$
\int_{1_{j}} S_{o y, j} \frac{d \varsigma}{\delta_{j}}, \int_{1_{j}} S_{o z, j} \frac{d \varsigma}{\delta_{j}} \text { and } \int_{1_{j}} S_{o \varpi, j} \frac{d \varsigma}{\delta_{j}} \quad=\sum_{\kappa=1}^{n_{g r i}}\left(\frac{l_{\kappa}^{\varsigma}}{6 \delta_{\kappa}^{\varsigma}}\left(S_{o y, \kappa}^{\varsigma, \text { start }}+4 S_{o y, \kappa}^{\varsigma, \text { mid }}+S_{o y, \kappa}^{\varsigma, \text { end }}\right)\right) ;
$$

- using following equations (3.9), (3.10) and (3.11) accordingly presented below,

$$
\begin{aligned}
& S_{h \sigma, j}=\int_{\ell_{r j}} \frac{S_{o \sigma, j}^{\varsigma}(\varsigma) d \varsigma}{\delta(\varsigma)}= \\
& =\sum_{\kappa=1}^{n_{\zeta i j}}\left(\frac{l_{\kappa}^{\varsigma}}{6 \delta_{\kappa}^{\varsigma}}\left(S_{o \sigma, \kappa}^{\varsigma, \text { start }}+4 S_{o \sigma, \kappa}^{\varsigma, \text { mid }}+S_{o \sigma, \kappa}^{\varsigma, \text { end }}\right)\right) .
\end{aligned}
$$

Let us define the following column vectors 
consisting of $n_{r}$ elements, $\forall j=\overline{1, n_{r}}$ (according to the number of edges of graph $\mathbf{G}$ ):

$$
\begin{gathered}
\vec{S}_{h z}=\left[\begin{array}{c}
S_{h z, 1} \\
S_{h z, 2} \\
\vdots \\
S_{h z, n_{r}}
\end{array}\right] ; \quad \vec{S}_{h y}=\left[\begin{array}{c}
S_{h y, 1} \\
S_{h y, 2} \\
\vdots \\
S_{h y, n_{r}}
\end{array}\right] ; \\
\vec{S}_{h \omega}=\left[\begin{array}{c}
S_{h \omega, 1} \\
S_{h \omega, 2} \\
\vdots \\
S_{h \omega, n_{r}}
\end{array}\right] ;
\end{gathered}
$$

Using weighting matrix of unbranched sectional parts W (2.2) introduced above as well as column vectors $\vec{S}_{h z}, \vec{S}_{h y}$ and $\vec{S}_{h \pi}$ presented above (3.12) we can rewrite Castigliano's functional (3.8) as the following vector-matrix equation:

$$
\begin{aligned}
& \mathbf{C}=\frac{1}{2 G} \stackrel{\stackrel{\mathrm{r}}{T}}{T} \mathbf{W} \stackrel{\mathrm{r}}{T_{S}}-\stackrel{\mathrm{r}}{T_{S}^{T}} \frac{Q_{y}}{G I_{z}} \stackrel{\mathrm{r}}{S_{h z}}- \\
& -T_{S}^{T} \frac{Q_{z}}{G I_{y}} \stackrel{\mathrm{r}}{S_{h y}}-\stackrel{\mathrm{r}}{T}_{S}^{T} \frac{M_{\sigma}}{G I_{\sigma}} \stackrel{\mathrm{r}}{S_{h \sigma}}+\mathrm{K}
\end{aligned}
$$

Next, for each section branch point we can work out equation of shear forces flows equilibrium in terms of projections on the longitudinal axis of the thin-walled bar. In order to obtain general view for these equations (the system of equations by the number of branch points in the section) we can use incidence matrixes $\dot{\mathbf{I}}$ and $|\dot{\mathbf{I}}|$ introduced above which reflect topological structure of considered cross-section of the thinwalled bar:

$$
(|\dot{\mathbf{I}}|+\dot{\mathbf{I}}) \vec{T}_{S}-(|\dot{\mathbf{I}}|-\dot{\mathbf{I}}) \vec{T}_{E}=\mathbf{0}
$$

where $\stackrel{\mathrm{r}}{T_{S}}=\left\{T_{S, j}\right\}^{T}, j=\overline{1, n_{r}}$ - vector of shear forces flows at start points of unbranched sectional parts; $T_{E}^{\mathrm{r}}=\left\{T_{E, j}\right\}^{T}, j=\overline{1, n_{r}}$ - vector of shear forces flows at end points of unbranched sectional parts:

$$
\stackrel{\mathrm{r}}{T_{E}}=\stackrel{\mathrm{r}}{T_{S}}-\Delta \stackrel{\mathrm{r}}{T}
$$

here $\Delta \stackrel{\mathrm{r}}{T}=\left\{\Delta T_{j}\right\}^{T}, j=\overline{1, n_{r}}$ - vector of shear forces flows increments for each unbranched sectional part:

$$
\Delta \stackrel{\mathrm{r}}{T_{j}}=\frac{Q_{y}}{I_{z}} \stackrel{\mathrm{r}}{S}_{z, j}+\frac{Q_{z}}{I_{y}} \stackrel{\mathrm{r}}{S_{y, j}}+\frac{M_{\varpi}}{I_{\varpi}} \stackrel{\mathrm{r}}{S_{\varpi, j}} ;
$$

where vectors $\stackrel{\mathrm{r}}{S}_{z, j},{\stackrel{\mathrm{r}}{S_{y, j}}}_{y},{\stackrel{\mathrm{r}}{S_{w, j}}}$ are presented below:

$$
\begin{gathered}
\vec{S}_{z}=\left[\begin{array}{c}
S_{z, 1} \\
S_{z, 2} \\
\vdots \\
S_{z, n_{r}}
\end{array}\right] ; \quad \vec{S}_{y}=\left[\begin{array}{c}
S_{y, 1} \\
S_{y, 2} \\
\vdots \\
S_{y, n_{r}}
\end{array}\right] ; \\
\vec{S}_{\sigma}=\left[\begin{array}{c}
S_{\sigma, 1} \\
S_{\sigma, 2} \\
\vdots \\
S_{\sigma, n_{r}}
\end{array}\right] ;
\end{gathered}
$$

and components of vectors $\stackrel{\mathrm{r}}{S}_{z, j}, \stackrel{\mathrm{r}}{S}_{y, j}, \stackrel{\mathrm{r}}{S}_{\varpi, j}$ can be calculated as follow, $\forall \kappa: \vec{s}_{\kappa}^{\varsigma} \in \mathbf{R}_{j}^{\varsigma} \wedge \vec{s}_{\kappa}^{\varsigma} \in \mathbf{S}^{\varsigma}$ :

$$
\begin{aligned}
& S_{z, j}=\int_{\ell_{r j}} y^{\varsigma}(\varsigma) \delta(\varsigma) d \varsigma= \\
& =\sum_{\kappa=1}^{n_{\zeta r i}}\left(\delta_{\kappa}^{\varsigma} l_{\kappa}^{\varsigma}\left(y_{\kappa}^{\varsigma, \text { start }}+\frac{1}{2} \Delta y_{\kappa}^{\varsigma}\right)\right) ; \\
& S_{y, j}=\int_{\ell_{r j}} z^{\varsigma}(\varsigma) \delta(\varsigma) d \varsigma= \\
& =\sum_{\kappa=1}^{n_{\zeta \zeta j}}\left(\delta_{\kappa}^{\varsigma} l_{\kappa}^{\varsigma}\left(z_{\kappa}^{\varsigma, \text { start }}+\frac{1}{2} \Delta z_{\kappa}^{\varsigma}\right)\right) ;
\end{aligned}
$$


Searching Shear Forces Flows for an Arbitrary Cross-Section of a Thin-Walled Bar: Development of Numerical Algorithm Based on the Graph Theory

$$
\begin{aligned}
& S_{\varpi, j}=\int_{\ell_{r j}} \varpi^{\varsigma}(\varsigma) \delta(\varsigma) d \varsigma= \\
& =\sum_{\kappa=1}^{n_{\zeta r i}}\left(\delta_{\kappa}^{\varsigma} l_{\kappa}^{\varsigma}\left(\varpi_{\kappa}^{\varsigma, \text { start }}+\frac{1}{2} \Delta \varpi_{\kappa}^{\varsigma}\right)\right) .
\end{aligned}
$$

Let us rewrite the equation (3.14) taken into account the equation (3.15), we obtain the following:

$$
\begin{gathered}
(|\dot{\mathbf{I}}|+\dot{\mathbf{I}}) \vec{T}_{S}-(|\mathbf{I}|-\dot{\mathbf{I}}) \times\left(\vec{T}_{S}-\Delta \vec{T}\right)=\mathbf{0} ; \\
(|\dot{\mathbf{I}}|+\dot{\mathbf{I}}) \vec{T}_{S}-(|\dot{\mathbf{I}}|-\dot{\mathbf{I}}) \vec{T}_{S}+(|\dot{\mathbf{I}}|-\dot{\mathbf{I}}) \Delta \vec{T}=\mathbf{0} \\
2 \dot{\mathbf{I}} \vec{T}_{S}+(|\dot{\mathbf{I}}|-\dot{\mathbf{I}}) \Delta \vec{T}=\mathbf{0} ;
\end{gathered}
$$

or taken into account the equation (3.16):

$$
\begin{aligned}
& 2 \dot{\mathbf{I}} \vec{T}_{S}+(|\dot{\mathbf{I}}|-\dot{\mathbf{I}}) \times \\
& \times\left(\frac{Q_{y}}{I_{z}} \vec{S}_{z, j}+\frac{Q_{z}}{I_{y}} \vec{S}_{y, j}+\frac{M_{\varpi}}{I_{\varpi}} \vec{S}_{\sigma, j}\right)=\mathbf{0} .
\end{aligned}
$$

The system of equation (3.21) has $n_{v}$ equilibrium equations. The last equation is linear-dependent or a linear combination from the previous equations. Let us rewrite the system of equations (3.21) excluding the last equilibrium equation:

$$
\begin{aligned}
& 2 \dot{\mathbf{I}}^{\prime} \vec{T}_{S}+\left(\left|\dot{\mathbf{I}}^{\prime}\right|-\dot{\mathbf{I}}^{\prime}\right) \times \\
& \times\left(\frac{Q_{y}}{I_{z}} \vec{S}_{z, j}+\frac{Q_{z}}{I_{y}} \vec{S}_{y, j}+\frac{M_{\varpi}}{I_{\varpi}} \vec{S}_{\varpi, j}\right)=\mathbf{0}
\end{aligned}
$$

where $\dot{\mathbf{I}}^{\prime}$ - incidence matrix of the graph $\mathbf{G}^{\prime}$ truncated by the last row with dimensions

$$
\left(n_{v}-1\right) \times n_{r}, \dot{\mathbf{I}}^{\prime}=\left\{g_{i j} \mid i=\overline{1, n_{v}-1}, j=\overline{1, n_{r}}\right\} ;
$$

$\left|\dot{\mathbf{I}}^{\prime}\right|$ - matrix composed using modulus of elements $g_{i j}$ of truncated matrix $\dot{\mathbf{I}}^{\prime}$ as

$$
\left|\dot{\mathbf{I}}^{\prime}\right|=\left\{\left|g_{i j}\right| \mid i=\overline{1, n_{v}-1}, j=\overline{1, n_{r}}\right\} .
$$

We also can work out the last equilibrium equation relating to the longitudinal axes $x-x$ of a thin-walled bar as condition of static equivalence of torsional moment caused by the shear forces flows to the free torsional moment $M_{x}$ acting in the cross-section of the thinwalled bar:

$$
M_{x}-\sum_{j=1}^{n_{r}} \int_{l_{j}} T_{j}(\varsigma) d \omega=0
$$

where $T_{j}(\varsigma)$ - shear forces flow at some point of the cross-section which can be expressed depending on shear forces flow $T_{S, j}(\varsigma)$ at start point of the corresponded unbranched part of the section as follow:

$$
T_{j}=T_{S, j}-\frac{Q_{y}}{I_{z}} S_{o z, j}-\frac{Q_{z}}{I_{y}} S_{o y, j}-\frac{M_{\varpi}}{I_{\varpi}} S_{o \varpi, j}
$$

here we omitted functional dependence from angular position $\varsigma$ (to simplify presented formulas).

Then:

$$
\begin{aligned}
& M_{x}-\sum_{j=1}^{n_{r}} \int_{1_{j}}\left(T_{S, j}-\frac{Q_{y}}{I_{z}} S_{o z, j}-\frac{Q_{z}}{I_{y}} S_{o y, j}-\frac{M_{\varpi}}{I_{\varpi}} S_{o \sigma, j}\right) \rho d \varsigma=0 ; \\
& M_{x}-\sum_{j=1}^{n_{r}}\left(T_{S, j} \int_{1_{j}} \rho d \varsigma-\frac{Q_{y}}{I_{z}} \int_{1_{j}} S_{o z, j} \rho d \varsigma-\frac{Q_{z}}{I_{y}} \int_{1_{j}} S_{o y, j} \rho d \varsigma-\frac{M_{\varpi}}{I_{\varpi}} \int_{1_{j}} S_{o \varpi, j} \rho d \varsigma\right)=0 .
\end{aligned}
$$


Finally, we've obtained [2]:

$$
\begin{gathered}
M_{x}-\sum_{j=1}^{n_{r}}\left(T_{S, j} \int_{1_{j}} \rho d \varsigma-\frac{Q_{y}}{I_{z}} \int_{1_{j}} S_{o z, j} \rho d \varsigma-\frac{Q_{z}}{I_{y}} \int_{1_{j}} S_{o y, j} \rho d \varsigma-\frac{M_{\varpi}}{I_{\varpi}} \int_{1_{j}} S_{o \varpi, j} \rho d \varsigma\right)=0 ; \\
\sum_{j=1}^{n_{r}} T_{S, j} \int_{1_{j}} \rho d \varsigma-\frac{Q_{y}}{I_{z}} \sum_{j=1}^{n_{r}} \int_{1_{j}} S_{o z, j} \rho d \varsigma-\frac{Q_{z}}{I_{y}} \sum_{j=1}^{n_{r}} \int_{1_{j}} S_{o y, j} \rho d \varsigma-\frac{M_{\varpi}}{I_{\varpi}} \sum_{j=1}^{n_{r}} \int_{1_{j}} S_{o \varpi, j} \rho d \varsigma-M_{x}=0
\end{gathered}
$$

here integrals

$$
\sum_{j=1}^{n_{r}} \int_{1_{j}} S_{o z, j} \rho d \varsigma, \sum_{j=1}^{n_{r}} \int_{1_{j}} S_{o y, j} \rho d \varsigma, \sum_{j=1}^{n_{r}} \int_{1_{j}} S_{o \sigma, j} \rho d \varsigma
$$

can be calculated using equations (3.26), (3.27) and (3.28) accordingly as presented below,

$$
\begin{aligned}
& \forall \kappa: \vec{s}_{\kappa}^{\varsigma} \in \mathbf{R}_{j}^{\varsigma} \wedge \vec{s}_{\kappa}^{\varsigma} \in \mathbf{S}^{\varsigma}: \\
& S_{\rho z}=\sum_{j=1}^{n_{r}} \int_{\ell_{r j}} S_{o z, j}^{\varsigma}(\omega) \rho d \varsigma= \\
& =\sum_{j=1}^{n_{r}}\left(\sum_{\kappa=1}^{n_{\zeta \zeta j}} \frac{\Delta \omega_{\kappa}^{\varsigma}}{6}\left(S_{o z, \kappa}^{\varsigma, \text { start }}+4 S_{o z, \kappa}^{\varsigma, \text { mid }}+S_{o z, \kappa}^{\varsigma, \text { end }}\right)\right) \text {; } \\
& S_{\rho y}=\sum_{j=1}^{n_{r}} \int_{\ell_{r j}} S_{o y, j}^{\varsigma}(\omega) \rho d \varsigma= \\
& =\sum_{j=1}^{n_{r}}\left(\sum_{\kappa=1}^{n_{c i j}} \frac{\Delta \omega_{\kappa}^{\varsigma}}{6}\left(S_{o y, \kappa}^{\varsigma, \text { start }}+4 S_{o y, \kappa}^{\varsigma, \text { mid }}+S_{o y, \kappa}^{\varsigma, \text { end }}\right)\right) ; \\
& S_{\rho \sigma}=\sum_{j=1}^{n_{r}} \int_{\ell_{r j}} S_{o \sigma, j}^{\varsigma}(\omega) \rho d \varsigma= \\
& =\sum_{j=1}^{n_{r}}\left(\sum_{\kappa=1}^{n_{\zeta j j}} \frac{\Delta \omega_{\kappa}^{\varsigma}}{6}\left(S_{o \varpi, \kappa}^{\varsigma, s t a r t}+4 S_{o \sigma, \kappa}^{\varsigma, \text { mid }}+S_{o \varpi, \kappa}^{\varsigma, \text { end }}\right)\right)
\end{aligned}
$$

Let us rewrite the constraints-equality (3.25) using vector representation taken into account equations (3.26), (3.27) and (3.28) as presented below:

$$
\begin{aligned}
& \stackrel{\mathrm{r}^{T}}{{ }^{T}} T_{S}-\frac{Q_{y}}{I_{z}} S_{\rho z}-\frac{Q_{z}}{I_{y}} S_{\rho y}- \\
& -\frac{M_{\varpi}}{I_{\varpi}} S_{\rho \sigma}-M_{x}=0 .
\end{aligned}
$$

Thus, the formulated problem is presented as mathematical programming task, namely as searching of unknown values of shear forces flows at start points of unbranched parts of a section:

$$
\stackrel{\mathrm{r}}{T_{S}}=\left\{T_{S, j}\right\}^{T}, j=\overline{1, n_{r}}
$$

which ensure the least value of the following Castigliano's functional C (3.13):

$$
\begin{aligned}
& \mathbf{C}=\frac{1}{2 G} \stackrel{\stackrel{\mathrm{r}}{T}}{T_{S}^{T}} \mathbf{W} \stackrel{\mathrm{r}}{T_{S}}-\stackrel{\mathrm{r}}{T_{S}^{T}} \frac{Q_{y}}{G_{z}} \stackrel{\stackrel{\mathrm{r}}{S}}{h z}- \\
& -\stackrel{\mathrm{r}}{T}_{S}^{T} \frac{Q_{z}}{G I_{y}} \stackrel{\mathrm{r}}{S_{h y}}-\stackrel{\mathrm{r}}{T_{S}^{T}} \frac{M_{\sigma}}{G I_{\sigma}} \stackrel{\stackrel{\mathrm{r}}{S}}{S_{h \sigma}}+\mathrm{K} \rightarrow \text { min }
\end{aligned}
$$

subject to the following equilibrium conditions (3.22) and (3.29):

$$
\left\{\begin{array}{l}
2 \dot{\mathbf{I}}^{\prime} \vec{T}_{S}+\left(\left|\dot{\mathbf{I}}^{\prime}\right|-\dot{\mathbf{I}}^{\prime}\right)\left(\frac{Q_{y}}{I_{z}} \vec{S}_{z, j}+\frac{Q_{z}}{I_{y}} \vec{S}_{y, j}+\frac{M_{\varpi}}{I_{\varpi}} \vec{S}_{\bar{\sigma}, j}\right)=\mathbf{0} ; \\
\vec{\omega}^{T} \vec{T}_{S}-\frac{Q_{y}}{I_{z}} S_{\rho z}-\frac{Q_{z}}{I_{y}} S_{\rho y}-\frac{M_{\sigma}}{I_{\sigma}} S_{\rho \sigma}-M_{x}=0 .
\end{array}\right.
$$


Searching Shear Forces Flows for an Arbitrary Cross-Section of a Thin-Walled Bar:

Development of Numerical Algorithm Based on the Graph Theory

Method of Lagrange multipliers can be used to point of the following modified reduce the mathematical programming task functional $\Lambda\left(\stackrel{\mathrm{r}}{T_{S}}, \stackrel{\mathrm{r}}{T}^{T}, \lambda_{n_{v}}\right)$ :
$(3.30)-(3.32)$ to the searching of stationary

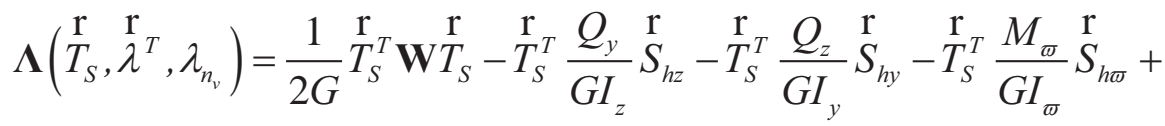

$$
\begin{aligned}
& +\stackrel{\mathrm{r}}{\lambda}^{T}\left[2 \dot{\mathbf{I}}^{\prime} \stackrel{\mathrm{r}}{S}_{S}+\left(\left|\dot{\mathbf{I}}^{\prime}\right|-\dot{\mathbf{I}}^{\prime}\right)\left(\frac{Q_{y}}{I_{z}} \stackrel{\mathrm{r}}{S_{z, j}}+\frac{Q_{z}}{I_{y}} \stackrel{\mathrm{r}}{S_{y, j}}+\frac{M_{\sigma}}{I_{\sigma}} \stackrel{\mathrm{r}}{S_{\sigma, j}}\right)\right]+ \\
& +\lambda_{n_{v}}\left[\stackrel{\mathrm{r}}{\omega}^{T} T_{S}^{\mathrm{r}}-\frac{Q_{y}}{I_{z}} S_{\rho z}-\frac{Q_{z}}{I_{y}} S_{\rho y}-\frac{M_{\varpi}}{I_{\sigma}} S_{\rho \sigma}-M_{x}\right] \rightarrow \min .
\end{aligned}
$$

here

$$
\stackrel{\mathrm{r}}{\lambda}=\left\{\lambda_{f}\right\}, f=\overline{1, n_{v}-1}
$$

Stationary conditions of the modified functional $\Lambda\left(\stackrel{r}{T_{S}}, \stackrel{r}{\lambda}{ }^{T}, \lambda_{n_{v}}\right)$ (3.33) can be transformed into the system of $n_{r}+n_{v}$ linear algebraic equations - vector of Lagrange multipliers consisting of $n_{v}-1$ elements; $\lambda_{n_{v}}-$ additional Lagrange and presented using vector-matrix form as follow [2]:

multiplier.

$$
\begin{aligned}
& \left\|\left[\begin{array}{ccc}
\frac{1}{G} \mathbf{W} & 2 \dot{\mathbf{I}}^{T} & \boldsymbol{\Delta} \boldsymbol{\omega}_{\mathbf{r}}^{\varsigma} \\
2 \dot{\mathbf{I}}^{\prime} & \boldsymbol{\Theta}_{n_{v}-1, n_{v}-1} & \mathbf{0}_{n_{v}-1} \\
\left(\Delta \boldsymbol{\omega}_{\mathbf{r}}^{\varsigma}\right)^{T} & \mathbf{0}_{n_{v}-1}^{T} & 0
\end{array}\right]|\times|\left[\begin{array}{c}
\vec{T}_{S} \\
\vec{\lambda} \\
\lambda_{n_{v}}
\end{array}\right]=M_{x} \times\right\|\left[\begin{array}{c}
\mathbf{0}_{n_{r}} \\
\mathbf{0}_{n_{v}-1} \\
1
\end{array}\right] \mid+\frac{Q_{y}}{I_{z}} \times \|\left[\begin{array}{c}
\frac{\vec{S}_{h z}}{G} \\
\left(\dot{\mathbf{I}}^{\prime}-\left|\dot{\mathbf{I}}^{\prime}\right|\right) \vec{S}_{z} \\
S_{\rho z}
\end{array}\right]+ \\
& +\frac{Q_{z}}{I_{y}} \times \llbracket\left(\begin{array}{c}
\frac{\vec{S}_{h y}}{G} \\
\left(\dot{\mathbf{I}}^{\prime}-\left|\dot{\mathbf{I}}^{\prime}\right|\right) \vec{S}_{y} \\
S_{\rho y}
\end{array}\right]+\frac{M_{\sigma}}{I_{\sigma}} \times \|\left(\begin{array}{c}
\frac{\vec{S}_{h \sigma}}{G} \\
\left(\dot{\mathbf{I}}^{\prime}-\left|\dot{\mathbf{I}}^{\prime}\right|\right) \vec{S}_{\sigma} \\
S_{\rho \sigma}
\end{array}\right]
\end{aligned}
$$

where:

$$
\mathbf{M}=\left[\begin{array}{ccc}
1 / G \mathbf{W} & 2 \dot{\mathbf{I}}^{T} & \boldsymbol{\Delta} \boldsymbol{\omega}_{\mathbf{r}}^{\varsigma} \\
2 \dot{\mathbf{I}}^{\prime} & \boldsymbol{\Theta}_{n_{v}-1, n_{v}-1} & \mathbf{0}_{n_{v}-1} \\
\left(\Delta \boldsymbol{\omega}_{\mathbf{r}}^{\varsigma}\right)^{T} & \mathbf{0}_{n_{v}-1}^{T} & 0
\end{array}\right]
$$


M - square matrix with dimensions $\left(n_{r}+n_{v}\right) \times\left(n_{r}+n_{v}\right)$, here $n_{r}$ and $n_{v}$ - number of edges and vertices of the graph $\mathbf{G}$ accordingly; $\Delta \omega_{\mathrm{r}}^{\varsigma}-$ column vector of sectorial coordinates increments

$$
\Delta \omega_{\mathbf{r}}^{\varsigma}=\left\{\Delta \omega_{r, j}^{\varsigma} \mid j=\overline{1, n_{r}}\right\}^{T}
$$

consisting of $n_{r}$ components calculated according to (2.3); $\vec{S}_{y}, \vec{S}_{z}, \vec{S}_{\sigma}-$ column vectors (3.17) with $n_{r}$ components calculated according to (3.18), (3.19) and (3.20) respectively; $\vec{S}_{h y}, \vec{S}_{h z}, \vec{S}_{h \varpi}-$ column vectors (3.12) with $n_{r}$ components calculated according to (3.9), (3.10) and (3.11) respectively; $S_{\rho y}$, $S_{\rho z}, S_{\rho \sigma}$ - integral section properties calculated according to (3.26), (3.27) and (3.28) respectively.

Solution of the system of equations (3.34) determines column vector of shear forces flows

$$
\stackrel{\mathrm{r}}{T_{S}}=\left\{T_{S, j}\right\}^{T}, j=\overline{1, n_{r}},
$$

at the start points of unbranched cross-section parts. The vector $\stackrel{\mathrm{r}}{T}_{S}$ can be also presented as follow:

$\stackrel{\mathrm{r}}{T_{S}}=M_{x} \mathrm{r}_{x}+\frac{Q_{y}}{I_{z}} \stackrel{\mathrm{r}}{b_{z}}+\frac{Q_{z}}{I_{y}} \stackrel{\mathrm{r}}{b_{y}}+\frac{M_{\varpi}}{I_{\varpi}} \mathrm{r}_{\varpi}$.

In this case the system of algebraic equations (3.34) disintegrates and transforms into the four systems of $n_{r}+n_{v}$ algebraic equations relating to the column vectors $\stackrel{\mathrm{r}}{b_{x}}, \stackrel{\mathrm{r}}{b}, \stackrel{\mathrm{r}}{b_{z}}$ and $\stackrel{\mathrm{r}}{b}$ consisting of $n_{r}$ elements [2] as presented below:

$$
\mathbf{M} \times \llbracket\left[\begin{array}{c}
\vec{b}_{x} \\
\vec{\lambda}_{x} \\
\lambda_{n_{v} x}
\end{array}\right]=\mid\left[\begin{array}{c}
\mathbf{0}_{n_{r}} \\
\mathbf{0}_{n_{v}-1} \\
1
\end{array}\right] ;
$$$$
\mathbf{M} \times\left\|\left[\begin{array}{c}
\vec{b}_{y} \\
\vec{\lambda}_{y} \\
\lambda_{n_{v} y}
\end{array}\right]=\right\|\left[\begin{array}{c}
\frac{\vec{S}_{h y}}{G} \\
\left(\dot{\mathbf{I}}^{\prime}-\left|\dot{\mathbf{I}}^{\prime}\right|\right) \times \vec{S}_{y} \\
S_{\rho y}
\end{array}\right] ;
$$$$
\mathbf{M} \times \llbracket\left[\begin{array}{c}
\vec{b}_{z} \\
\vec{\lambda}_{z} \\
\lambda_{n_{v} z}
\end{array}\right]=\llbracket\left(\begin{array}{c}
\vec{S}_{h z} \\
G \\
\left(\dot{\mathbf{I}}^{\prime}-\left|\dot{\mathbf{I}}^{\prime}\right|\right) \times \vec{S}_{z} \\
S_{\rho z}
\end{array}\right] ;
$$

$$
\mathbf{M} \times\left\lfloor\left[\begin{array}{c}
\vec{b}_{\sigma} \\
\vec{\lambda}_{\sigma} \\
\lambda_{n_{v} \sigma}
\end{array}\right]=\llbracket\left[\begin{array}{c}
\frac{\vec{S}_{h \sigma}}{G} \\
\left(\dot{\mathbf{I}}^{\prime}-\left|\dot{\mathbf{I}}^{\prime}\right|\right) \times \vec{S}_{\sigma} \\
S_{\rho \sigma}
\end{array}\right] ;\right.
$$

where

$$
\begin{gathered}
\vec{\lambda}_{x}=\left\{\lambda_{x, f}\right\}^{T}, \vec{\lambda}_{y}=\left\{\lambda_{y, f}\right\}^{T}, \vec{\lambda}_{z}=\left\{\lambda_{z, f}\right\}^{T}, \\
\vec{\lambda}_{\sigma}=\left\{\lambda_{\sigma, f}\right\}^{T}, f=\overline{1, n_{v}-1}
\end{gathered}
$$

- unknown column vectors of Lagrange multipliers consisting of $n_{v}-1$ elements;

$$
\lambda_{n_{v} x}, \lambda_{n_{v} y}, \lambda_{n_{v} z}, \lambda_{n_{v} \sigma}
$$

- additional Lagrange multipliers.

Projection of the vector

$$
\vec{b}_{x}=\left\{b_{x, j} \mid j=\overline{1, n_{r}}\right\}
$$

defined of the set of $n_{r}$ unbranched sectional parts into the set of sectional segments 
Searching Shear Forces Flows for an Arbitrary Cross-Section of a Thin-Walled Bar: Development of Numerical Algorithm Based on the Graph Theory

$$
\vec{b}_{x}^{\varsigma}=\left\{b_{x, \kappa}^{\varsigma} \mid \kappa=\overline{1, n_{\varsigma}-1}\right\}
$$

can be written as:

$$
b_{x, \kappa}^{\varsigma}=b_{x, j} \forall \kappa: \vec{s}_{\kappa}^{\varsigma} \subseteq \mathbf{R}_{j}^{\varsigma}
$$

and

$$
b_{x, \kappa}^{\varsigma}=0 \quad \forall \kappa: \vec{s}_{\kappa}^{\varsigma} \cap \mathbf{R}_{j}^{\varsigma}=\varnothing .
$$

By analogy column vectors

$$
\vec{b}_{y}=\left\{b_{y, j} \mid j=\overline{1, n_{r}}\right\}, \vec{b}_{z}=\left\{b_{z, j} \mid j=\overline{1, n_{r}}\right\}
$$

and $\vec{b}_{\varpi}=\left\{b_{\varpi, j} \mid j=\overline{1, n_{r}}\right\}$ can be also projected into the set of sectional segments obtaining corresponded column vectors

$$
\begin{aligned}
& \vec{b}_{y}^{\varsigma}=\left\{b_{y, \kappa}^{\varsigma} \mid \kappa=\overline{1, n_{\varsigma}-1}\right\}, \\
& \vec{b}_{z}^{\varsigma}=\left\{b_{z, \kappa}^{\varsigma} \mid \kappa=\overline{1, n_{\varsigma}-1}\right\}, \\
& \vec{b}_{\varpi}^{\varsigma}=\left\{b_{\varpi, \kappa}^{\varsigma} \mid \kappa=\overline{1, n_{\varsigma}-1}\right\} .
\end{aligned}
$$

The following transformations for the first moments of inertia and for the sectorial moment of inertia should be performed, $\forall \kappa=\overline{1, n_{\varsigma}-1}$ :

$$
\begin{gathered}
\bar{S}_{o z, \kappa}^{\varsigma} \leftarrow\left\{S_{o z, \kappa}^{\varsigma}-b_{z, \kappa}^{\varsigma}\right\} ; \\
\bar{S}_{o y, \kappa}^{\varsigma} \leftarrow\left\{S_{o y, \kappa}^{\varsigma}-b_{y, \kappa}^{\varsigma}\right\} ; \\
\bar{S}_{o \varpi, \kappa}^{\varsigma} \leftarrow\left\{S_{o \varpi, \kappa}^{\varsigma}-b_{\varpi, \kappa}^{\varsigma}\right\} ; \\
\tilde{S}_{o \varpi, \kappa}^{\varsigma} \leftarrow\left\{\bar{S}_{o \varpi, \kappa}^{\varsigma}-a_{\kappa}^{\varsigma} \frac{I_{\varpi}}{\Omega_{0}}\right\} .
\end{gathered}
$$

\section{SHEAR FORCES FLOWS AND SHEAR STRESSES}

Let us define the sets of shear forces flows values for the start, middle and end points at the middle line of the sectional segments

$$
\begin{gathered}
\mathbf{T}^{\varsigma, \text { st }}=\left\{T_{\kappa}^{\varsigma, \text { st }}\right\}, \mathbf{T}^{\varsigma, \text { mid }}=\left\{T_{\kappa}^{\varsigma, \text { mid }}\right\}, \\
\mathbf{T}^{\varsigma, \text { end }}=\left\{T_{\kappa}^{\varsigma, \text { end }}\right\}, \kappa=\overline{1, n_{\varsigma}-1},
\end{gathered}
$$

consisting of $n_{\varsigma}-1$ elements (by the number of sectional segments) as presented below [5]:

$$
\begin{aligned}
& T_{\kappa}^{\varsigma, \text { start }}=\frac{\wp H}{\Omega_{0}} a_{\kappa}^{\varsigma}-\frac{Q_{y}}{I_{z}} \bar{S}_{o z, \kappa}^{\varsigma, \text { start }}- \\
& -\frac{Q_{z}}{I_{y}} \bar{S}_{o y, \kappa}^{\varsigma, \text { start }}-\frac{M_{\varpi}}{I_{\varpi}} \tilde{S}_{o \sigma, \kappa}^{\varsigma, \text { start }} ; \\
& T_{\kappa}^{\varsigma, \text { mid }}=\frac{\wp H}{\Omega_{0}} a_{\kappa}^{\varsigma}-\frac{Q_{y}}{I_{z}} \bar{S}_{o z, \kappa}^{\varsigma, \text { mid }}- \\
& -\frac{Q_{z}}{I_{y}} \bar{S}_{o y, \kappa}^{\varsigma, \text { mid }}-\frac{M_{\sigma}}{I_{\varpi}} \tilde{S}_{o \sigma, \kappa}^{\varsigma, \text { mid }} ; \\
& T_{\kappa}^{\varsigma, \text { end }}=\frac{\wp H}{\Omega_{0}} a_{\kappa}^{\varsigma}-\frac{Q_{y}}{I_{z}} \bar{S}_{o z, \kappa}^{\varsigma, \text { end }}- \\
& -\frac{Q_{z}}{I_{y}} \bar{S}_{o y, \kappa}^{\varsigma, \text { end }}-\frac{M_{\sigma}}{I_{\varpi}} \tilde{S}_{o \sigma, \kappa}^{\varsigma, \text { end }} ;
\end{aligned}
$$

here the first moments of inertia $\bar{S}_{o z, \kappa}^{\varsigma}, \bar{S}_{o y, \kappa}^{\varsigma}$ and sectorial moment of inertia $\tilde{S}_{\text {oซ, } \kappa}^{\varsigma}$ should be calculated using transformations (3.37) and (3.38) accordingly.

Shear stresses for each $\kappa^{\text {th }}$ sectional segment

$$
\boldsymbol{\tau}^{\varsigma}=\left\{\vec{\tau}_{\kappa}^{\varsigma}=\left\{\tau_{\kappa}^{\varsigma, \text { start }}, \tau_{\kappa}^{\varsigma, \text { mid }}, \tau_{\kappa}^{\varsigma, \text { end }}\right\}\right\}, \kappa=\overline{1, n_{\varsigma}-1}
$$

can be calculated as presented below:

$$
\boldsymbol{\tau}_{\kappa}^{\varsigma}=\left\{\begin{array}{l}
\tau_{\kappa}^{\varsigma, \text { start }}=\left|\frac{T_{\kappa}^{\varsigma, \text { start }}}{\delta_{\kappa}^{\varsigma}}\right| \pm \frac{(1-\wp) H \delta_{\kappa}^{\varsigma}}{I_{k}} \\
\tau_{\kappa}^{\varsigma, \text { mid }}=\left|\frac{T_{\kappa}^{\varsigma, \text { mid }}}{\delta_{\kappa}^{\varsigma}}\right| \pm \frac{(1-\wp) H \delta_{\kappa}^{\varsigma}}{I_{k}} \\
\tau_{\kappa}^{\varsigma, \text { end }}=\left|\frac{T_{\kappa}^{\varsigma, \text { end }}}{\delta_{\kappa}^{\varsigma}}\right| \pm \frac{(1-\wp) H \delta_{\kappa}^{\varsigma}}{I_{k}}
\end{array}\right\},
$$

here torsion moment of inertia $I_{x}$ and parameter 
$\wp$ should be calculated as:

$$
\begin{gathered}
I_{x}=I_{k}+I_{\Gamma}=\frac{1}{3} \sum_{\kappa=1}^{n_{\varsigma}-1} l_{\kappa}^{\varsigma}\left(\delta_{\kappa}^{\varsigma}\right)^{3}+I_{\Gamma} ; \\
\wp=1-\frac{I_{k}}{I_{x}} ;
\end{gathered}
$$

and components

$$
\left|\frac{T_{\kappa}^{\zeta, \text { start }}}{\delta_{\kappa}^{\varsigma}}\right|,\left|\frac{T_{\kappa}^{\varsigma, \text { mid }}}{\delta_{\kappa}^{\varsigma}}\right| \text { and }\left|\frac{T_{\kappa}^{\zeta, \text { end }}}{\delta_{\kappa}^{\varsigma}}\right|
$$

in equations (4.4) define shear stresses values for start, middle and end points at the middle line of $\kappa^{\text {th }}$ sectional segment accordingly. Besides, transition from the shear stresses related to the middle line of $\kappa^{\text {th }}$ segment to the shear stresses at the outside longitudinal edges of this segment can be performed by addition or subtraction of the member

$$
\frac{(1-\wp)}{I_{k}} H \delta_{\kappa}^{\varsigma} .
$$

\section{CONSLUSIONS}

Searching problem of shear stresses on outside longitudinal edges of an arbitrary cross-section (including open-closed multi-contour crosssections) of a thin-walled bar subjected to the general load case has been considered in the paper. Formulated problem has been transformed into minimization problem of Castigliano's functional subject to constraintsequalities of shear forces flows equilibrium formulated for cross-section branch points as well as subject to equilibrium equation for the whole cross-section relating to longitudinal axes of the thin-walled bar.

Detail numerical algorithm intended to solve the formulated problem using mathematical apparatus of the graph theory has been proposed by the paper. The algorithm is oriented on software implementation in systems of computer- aided design of thin-walled bar structures.

\section{REFERENCES}

1. Perelmuter A.V., Slivker V.I. Raschetnie Modeli Soorujenii i Vozmojnost ih Analiza [Design Models of Structures and Analysis Possibilities]. Moscow, Publishers DMK, 2007, 595 pages (in Russian).

2. Slivker V.I. Stroitelnaya Mehanika. Variacionnie Osnovi [Structural mechanic. Variation Principles]. Moscow, Publishers ASV, 2005, 736 pages (in Russian).

3. Yurchenko V.V. Rozpodil Potokiv Dotichnih Zusil Vzdovj Zamknenih Konturiv Pererizu Tonkostinnogo Sterjnya: Rozrobka Chislovogo Algoritmu z Vikoristannyam Teoriï Grafiv [Distribution of the Shear Forces Flows Along the Closed Contours of a Section of a Thin-Walled Bar: Development of Numerical Algorithm Based on the Graph Theory]. // Resursoekonomni Materiali, Konstrukciï, Budivli ta Sporudi [Resource-Economical Materials, Structures and Buildings]. Collection of Scientific Papers, Issue 30, Rivne, 2015. pp. 306-316.

4. Yurchenko V.V. Uzagalneni Sektorialni Koordinati Dlya Dovilnogo Pererizu Tonkostinnogo Sterjnya_ Rozrobka Chislovogo Algoritmu z Vikoristannyam Teoriï Grafiv [Normalized Sectorial Coordinates for an Arbitrary Section of a Thin-Walled Bar: Development of Numerica Algorithm based on the Graph Theory]. // Resursoekonomni Materiali, Konstrukciï, Budivli ta Sporudi [Resource-Economical Materials, Structures and Buildings]. // Collection of Scientific papers, Issue 31, Rivne, 2015, pp. 538-549.

5. Yurchenko V.V. Chislennoe Reshenie Zadachi o Raspredelenii Kasatelnih Napryajenii V Sechenii Tonkostennogo Sterjnya Proizvolnoi Konfiguracii [Numerical Solution for the Problem About Distribution of Shear Forces Flows for an 
Searching Shear Forces Flows for an Arbitrary Cross-Section of a Thin-Walled Bar:

Development of Numerical Algorithm Based on the Graph Theory

Arbitrary Section of a Thin-Walled Bar]. // Stroitelnaya Mehanika i Stroitelnie Konstrukcii [Structural Mechanic and Building Structures]: Collection of Papers. Moscow, Publishers SCAD SOFT, 2013, pp. 487-505 (in Russian).

6. Alfano G., Marotti de Sciarra F., Rosati L. Automatic Analysis of Multicell Thin-Walled Sections. // Computer and Structures, 1996, No. 59, pp. 641-655.

7. Paz M., Strehl C.P., Schrader P. Computer Determination of the Shear Center of Open and Closed Sections. // Computer and Structures, 1976, No. 6, pp. 117-125.

8. Perelmuter A.V., Slivker V. I. Numerical structural analysis: models, methods and pitfalls. - Berlin-Heidelberg-New YorkHong Kong-London-Milan-Paris-Tokyo: Springer Verlag, 2003, 600 pages.

9. Perelmuter A., Yurchenko V. Shear Stresses in Hybrid Thin-Walled Section: Development of Detail Numerical Algorithm Based on the Graph Theory. // Proceedings of 3rd Polish Congress of Mechanics and 21st International Conference on Computer Methods in Mechanics. Short Papers, Vol. 2, Gdańsk, 2015, pp. 943-944.

10. Prokić A. Computer Program for Determination of Geometrical Properties of Thin-Walled Beams with Open-Closed Section. // Computers and Structures, 2000, No. 74, pp. 705-715.

11. Tarjan R. Depth-First Search and Linear Graph Algorithms. // SIAM Journal Computing, 1972, No. 1, pp. 146-160.

12. Waldron P. Sectorial Properties of Straight Thin-Walled Beams. // Computers \& Structures, 1986, Vol. 24, Issue 1, pp. 147-156.

\section{СПИСОК ЛИТЕРАТУРЫ}

1. Перельмутер А.В.,

Сливкер В.И. Расчетные модели сооружений и возможность их анализа. - М.: ДМК Пресc, 2007. - 595 c.
2. Сливкер В.И. Строительная механика. Вариационные основы. - М.: АСВ, 2005. $-736 \mathrm{c}$.

3. Юрченко В.В. Розподіл потоків дотичних зусиль вздовж замкнених контурів перерізу тонкостінного стержня: розробка числового алгоритму 3 використанням теорії графів [Распределение потоков касательных усилий вдоль замкнутых контуров сечения тонкостенного стержня: разработка численного алгоритма с использованием теории графов]. // Ресурсоекономні матеріали, конструкції, будівлі та споруди [Ресурсоэкономные материалы, конструкции, здания и сооружения]. Сборник научных трудов. Выпуск 30, Ровно, 2015, с. 306-316.

4. Юрченко В.В. Узагальнені секторіальні координати для довільного перерізу тонкостінного стержня: розробка числового алгоритму 3 використанням теорії графів [Обобщенные секториальные координаты для произвольного сечения тонкостенного стержня: разработка численного алгоритма с использованием теории графов]. // Ресурсоекономні матеріали, конструкції, будівлі та споруди [Ресурсоэкономные материалы, конструкции, здания и сооружения]. Сборник научных трудов. Выпуск 31, Ровно, 2015, с. 538-549.

5. Юрченко В.В. Численное решение задачи о распределении касательных напряжений в сечении тонкостенного стержня произвольной конфигурации // Строительная механика и строительные конструкции: Сборник статей. - М.: SCAD Soft, 2013, c. 487-505.

6. Alfano G., Marotti de Sciarra F., Rosati L. Automatic Analysis of Multicell Thin-Walled Sections. // Computer and Structures, 1996, No. 59, pp. 641-655.

7. Paz M., Strehl C.P., Schrader P. Computer Determination of the Shear Center of Open and Closed Sections. // 
Computer and Structures, 1976, No. 6, pp. 117-125.

8. Perelmuter A.V., Slivker V. I. Numerical structural analysis: models, methods and pitfalls. - Berlin-Heidelberg-New YorkHong Kong-London-Milan-Paris-Tokyo: Springer Verlag, 2003. 600 p.

9. Perelmuter A., Yurchenko V. Shear Stresses in Hybrid Thin-Walled Section: Development of Detail Numerical Algorithm Based on the Graph Theory. // Proceedings of 3rd Polish Congress of Mechanics and 21st International Conference on Computer Methods in Mechanics. Short Papers, Vol. 2, Gdańsk, 2015, pp. 943-944.

10. Prokić A. Computer Program for Determination of Geometrical Properties of Thin-Walled Beams with Open-Closed Section. // Computers and Structures, 2000, No. 74, pp. 705-715.

11. Tarjan R. Depth-First Search and Linear Graph Algorithms. // SIAM Journal Computing, 1972, No. 1, pp. 146-160.

12. Waldron P. Sectorial Properties of Straight Thin-Walled Beams. // Computers \& Structures, 1986, Vol. 24, Issue 1, pp. 147-156.

Vitalina V. Yurchenko, Candidate of Technical Science, Associate Professor, Kyiv National University of Civil Engineering and Architecture, 03680, Povitroflotskyj avenue, 31, Kyiv, Ukraine;

E-mail: vitalina@scadsoft.com.

Юрченко Виталина Витальевна, кандидат технических наук, доцент, Киевский национальный университет строительства и архитектуры; 03680, Воздухофлотский проспект, 31, Киев, Украина;

E-mail: vitalina@scadsoft.com. 